\title{
Racial disparities in kidney transplant waitlist appearance in Chicago: Is it race or place?
}

\author{
Robert B. Peng ${ }^{1}$ | Haena Lee ${ }^{2}$ | Zheng T. Ke ${ }^{3}$ | Milda R. Saunders ${ }^{4}$ (D)
}

${ }^{1}$ Department of Biostatistics, University of Michigan, Ann Arbor, MI, USA

${ }^{2}$ Institute for Social Research, University of Michigan, Ann Arbor, MI, USA

${ }^{3}$ Department of Statistics, University of Chicago, Chicago, IL, USA

${ }^{4}$ Section of General Internal Medicine, Department of Medicine, University of Chicago Medicine, Chicago, IL, USA

\section{Correspondence}

Milda R. Saunders, Department of Medicine, University of Chicago Medicine, Chicago, IL, USA.

Email:msaunders@uchicago.edu

\section{Funding information}

National Institute of Diabetes and Digestive and Kidney Diseases, Grant/Award Number: NIDDK K23 DK103111

\begin{abstract}
Background: Prior work has demonstrated how neighborhood poverty and racial composition impact racial disparities in access to the deceased donor kidney transplant waitlist, both nationally and regionally. We examined the association between neighborhood characteristics and racial disparities in time to transplant waitlist in Chicago, a diverse city with continued neighborhood segregation.

Methods: Using data from the United States Renal Data System (USRDS) and the US Census, we investigated time from dialysis initiation to kidney transplant waitlisting for African American and white patients in Chicago using cause-specific proportional hazards analyses, adjusting for individual sociodemographic and clinical characteristics, as well as neighborhood poverty and racial composition.

Results: In Chicago, African Americans are significantly less likely than whites to appear on the renal transplant waitlist (HR 0.73, $P<.05)$. Compared to whites in nonpoor neighborhoods, African Americans in poor neighborhoods are significantly less likely to appear on the transplant waitlist (HR 0.61, $P<.05$ ). Over 69\% of African Americans with ESRD live in these neighborhoods.

Conclusions: Consistent with national data, African Americans in Chicago have a lower likelihood of waitlisting than whites. This disparity is explained in part by neighborhood poverty, which impacts the majority of African American ESRD patients in Chicago.
\end{abstract}

KEYWORDS

geographic factors, healthcare disparity, kidney transplant, urban health

\section{1 | INTRODUCTION}

For patients with end-stage renal disease (ESRD), kidney transplantation is associated with higher quality of life, lower healthcare expenditure, and lower mortality compared to dialysis. ${ }^{1,2}$ Racial disparities in access to kidney transplantation have been well documented, and the underlying causes of these disparities are diverse. $^{3-9}$ Disparities in access to kidney transplant due to patientlevel factors, such as age, education, income, insurance status, and socioeconomic status have all been investigated. ${ }^{3-7}$ In addition, disparities due to provider-level factors, such as physician bias, have also been observed. ${ }^{8}$ Receiving a kidney transplant is also influenced by a variety of biological factors, which may vary by race, including the availability of a compatible donor organ due to blood type and HLA match.

Time from dialysis initiation to transplant waitlist may serve as a better marker for access because it measures nonbiological social factors. ${ }^{10,11}$ Prior work has shown that racial disparities in transplant waitlist exist at the national and regional levels. ${ }^{9,10}$ In addition, it has been observed that geographical variation and neighborhood characteristics play an important role in access to the renal transplant waitlist. 9,10,12-14 
Our prior work examined national data across 11 different United Network for Organ Sharing (UNOS) geographic regions and found that African American patients were less likely than white patients to appear on the renal transplant waitlist, and the magnitude of the disparity varied by region. ${ }^{9}$ To date, racial disparities in transplant waitlist in smaller geographic areas have been less explored. Examining a particular city as a case study allows us to control for potentially confounding broader geographic characteristics. Moreover, large cities have racial and class diversity, while having common cultural, economic, and healthcare resources. We sought to examine racial disparities in renal transplant waitlist focusing only on patients in the city of Chicago, the most populous city in the Midwest. Chicago makes an ideal case study as it has a large and diverse ESRD population, multiple transplant centers, and continued neighborhood segregation by race, income, and class. ${ }^{15}$

\section{2 | PATIENTS AND METHODS}

\section{1 | Subjects and data}

We used data from the United States Renal Data System (USRDS), a comprehensive national data system containing information about individuals with end-stage renal disease (ESRD) in the USA. ${ }^{1}$ Our data included non-Hispanic white and non-Hispanic African American individuals between the ages of 18 and 70 who initiated dialysis between January 2005 and September 2009 and had ZIP codes indicating residence in Chicago. Our initial study population included a total of 3438 individuals. We excluded individuals who were missing the date of their first ESRD service (dialysis) or had a transplant date prior to the date of their first ESRD service $(n=194)$. The final study population then consisted of 3244 individuals. Sociodemographic and clinical information for these individuals was collected from the USRDS medical evidence form.

Using ZIP code as a proxy for neighborhood, we used the US Census Bureau's 2007-2011 American Community Survey 5-Year Estimates to obtain two neighborhood-level characteristics, percent of neighborhood under the Federal Poverty Level and percent of neighborhood composed of African Americans. ${ }^{16}$

\section{2 | Variables}

\subsection{1 | Outcome variable}

The main outcome variable was time (in days) from dialysis initiation to appearance on the transplant waitlist, subject to censoring. Patients were censored at time of living donor transplant and if they were not listed on the waitlist by the end of the observation period, 10/1/2010. In addition, death was treated as a competing risk for waitlist appearance. Of the 2713 patients (83.6\%) who were not waitlisted during the observation period, 889 (27.4\%) died prior to waitlist appearance, 22 $(0.7 \%)$ received a living donor transplant prior to waitlist appearance, and 1802 (55.6\%) were not waitlisted by the end of the observation period (and did not die or receive a living donor transplant). See Flowchart.

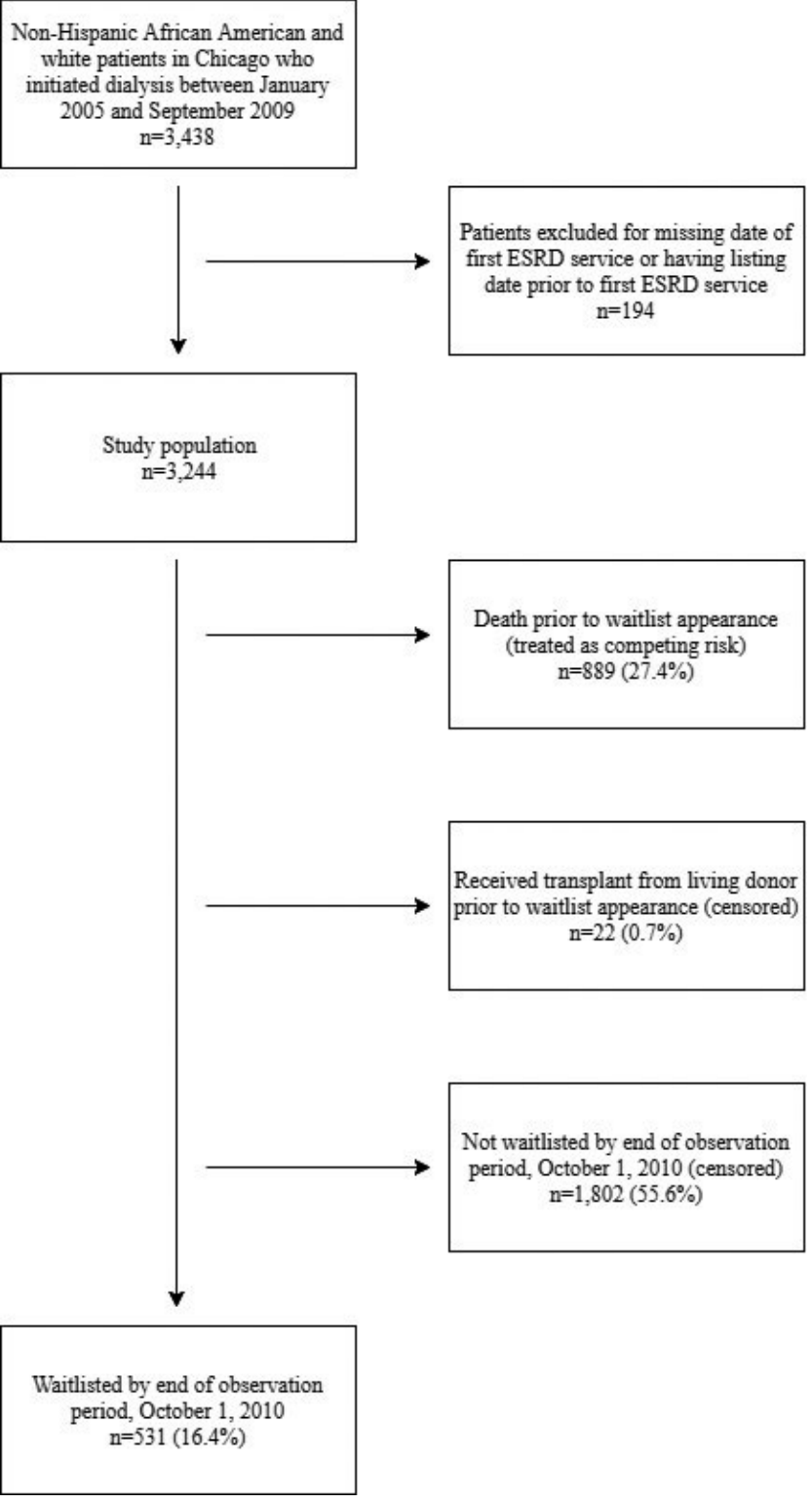

FLOWCHART: How the study population was derived and possible patient outcomes, including censored patients and patients experiencing competing risk of death

\subsection{2 | Explanatory variables}

The primary explanatory variable of interest is the patient's race, coded as an indicator variable, denoting African American (1) and white (0). Using guidance from prior literature, we identified 11 other sociodemographic and clinical characteristics (8 categorical and 3 continuous) as important covariates. ${ }^{14,17}$ These variables are sex, medical insurance coverage, employment status, hypertension, diabetes, coronary artery disease, cancer, inability to ambulate, age, body mass index, and hemoglobin level. While most of the categorical variables are binary, employment status and insurance status have multiple categories. Employment categories were collapsed into a binary variable, representing unemployment or another employment status, as unemployment has been shown to restrict access to renal transplantation. ${ }^{18}$ 
For insurance status, we categorized primary insurance as (Uninsured, Medicaid, Medicare, private insurance, and other insurance status) based on prior work. ${ }^{19}$ Uninsured and other insurance status contain individuals whose primary insurance status may not have been established (they could be going through the process of applying for Medicare, etc.) at time the Medical Evidence form was completed.

Of the study population, 335 patients (10.3\%) either did not have a value listed or lacked sufficient information to calculate their hemoglobin count. For those with missing hemoglobin values, our method of imputation was to replace each patient's missing value with the median hemoglobin measure of their race and sex cohort. Then, we created an additional indicator variable identifying the patients that were missing a hemoglobin measure, and this indicator variable was also included as a covariate. Similarly, for the 216 patients (6.7\%) with missing BMI, we imputed the missing values with the median of their race and sex cohort. Another indicator variable identifying these patients was included as a covariate.

In addition, using ZIP codes as a proxy for neighborhoods, we accounted for two neighborhood-level characteristics, the percent of a ZIP code living under the Federal Poverty Level and the percent of a ZIP code comprising non-Hispanic African Americans. Based on US Census definitions, poor neighborhoods (POOR) were defined as neighborhoods with $\geq 20 \%$ of individuals in the zip code below poverty and those with $<20 \%$ of individuals below poverty were considered nonpoor (RICH). ${ }^{20}$ Predominantly African American neighborhoods (AA) were defined as those with $\geq 50 \%$ of the ZIP code as African American, and non-African American neighborhoods (WHITE) were those with ZIP codes $<50 \%$ African American. We combined these two variables into one categorical variable, which included the following categories: (i) neighborhoods with less than $20 \%$ of the population below poverty and less than 50\% African American population (RICH, WHITE); (ii) neighborhoods with less than $20 \%$ of the population below poverty and greater than $50 \%$ African American population ( $\mathrm{RICH}, \mathrm{AA}$ ); (iii) neighborhoods with greater than $20 \%$ of the population below poverty and $<50 \%$ African American population (POOR, WHITE); and (iv) neighborhoods with $20 \%$ of the population below poverty and $>50 \%$ African American population (POOR, AA).

\section{3 | Analysis}

The primary outcome variable of interest was time to kidney transplant waitlist appearance, that is the number of days from the date of dialysis initiation to the date when the patient was listed on the deceased donor kidney transplant waitlist. Individuals were censored at time of living donor transplant and if they were not waitlisted by the end of the observation period, 10/1/2010. In addition, due to the relatively large proportion of deaths (27.4\%) prior to waitlist appearance, we treated death as a competing risk for waitlist appearance.

To test whether there was a significant difference between African American and white patients with respect to the other covariates, we used a Pearson's chi-square test for categorical variables and a Wilcoxon rank-sum test for quantitative variables. This was also done to test for significant differences between patients who were waitlisted vs not waitlisted. A $P$-value of $<.05$ was considered statistically significant.

We first looked at univariable survival analysis models before proceeding to build multivariable models. Due to the presence of competing risks, Kaplan-Meier curves are unreliable, so we instead used cumulative incidence competing risk (CICR) methods to give estimates of the cumulative incidence function for each of our categorical covariates, which can be found in Appendix 1. For the univariable analysis of continuous covariates, we instead fit a univariable Fine and Gray-based competing-risks regression and reported the $P$-values for each continuous coefficient (also in Appendix 1).

For the multivariable analysis, we constructed a proportional cause-specific hazards model based on time to renal transplant waitlisting. ${ }^{21}$ We first constructed a Cox model with race as our primary explanatory variable of interest, adjusting for sociodemographic (age, sex, insurance coverage, and employment status) and clinical characteristics (BMI, hemoglobin measure, and the presence of diabetes, hypertension, coronary artery disease, cancer, and inability to ambulate). Due to the strong interaction between individual race and neighborhood, we then created a race by neighborhood interaction term with eight categories, combining individual race with every category of the neighborhood advantage variable. We constructed a second Cox model with this aggregate variable, adjusting for sociodemographic and clinical characteristics. To control for correlation, we used robust estimates of variance with ZIP code as a cluster variable.

After we built our multivariable Cox models with either race or the race by neighborhood interaction term and the other covariates (as well as the indicator variables representing missing values for hemoglobin and BMI), we used the Wald chi-square test to test for the significance of the model as a whole. Because our final models included categorical variables, we calculated the $\mathrm{C}$-statistic to get an estimate of the predictive power of our models. Tests of the proportional hazards assumption of the Cox model (based on nonzero slope of Schoenfeld residuals) were also conducted. The goodness of fit of the models was also assessed using Cox-Snell residuals (Appendix 2). To test for bias introduced by our missing data, we added interactions between the dummy variables and the variables with missing data (BMI and hemoglobin level).

To examine how much of racial disparities are explained by neighborhood poverty and racial composition, we compared our first model with individual race and no neighborhood-level covariates to a new model with race and our neighborhood variable as separate predictors. To quantify the proportion of the racial disparity in hazard of kidney transplant waitlist appearance explained by neighborhood poverty and racial composition, we used the simple estimate $\left(\mathrm{HR}_{\text {new }}-\mathrm{HR}_{\text {original }}\right) /$ $\left(1-H R_{\text {original }}\right) .^{22}$

As an exploratory measure, we also constructed Cox models using only a race by neighborhood poverty interaction or a race by neighborhood racial composition interaction (along with the other sociodemographic and clinical characteristics). To further examine if neighborhood impacts African American and white patients differently, we constructed separate Cox models stratified by individual race 


\begin{tabular}{|c|c|c|c|}
\hline & $\begin{array}{l}\text { Overall } \\
\text { (3244 patients) }\end{array}$ & $\begin{array}{l}\text { Waitlisted } \\
\text { (531 patients) }\end{array}$ & $P$-value \\
\hline African American race & 2690 (82.9\%) & 440 (82.8\%) & .968 \\
\hline Male sex & 1808 (55.7\%) & $295(55.6 \%)$ & .928 \\
\hline \multicolumn{4}{|l|}{ Primary medical insurance } \\
\hline Uninsured & $541(16.7 \%)$ & 102 (19.2\%) & \multirow[t]{5}{*}{$<.001^{*}$} \\
\hline Medicaid & $1063(32.8 \%)$ & $131(24.7 \%)$ & \\
\hline Medicare & $576(17.8 \%)$ & $57(10.7 \%)$ & \\
\hline Private insurance & $829(25.6 \%)$ & $208(39.2 \%)$ & \\
\hline Other insurance status & $235(7.2 \%)$ & $33(6.2 \%)$ & \\
\hline Unemployed & 1203 (37.1\%) & $166(31.3 \%)$ & $.002^{*}$ \\
\hline Hypertension presence & 2737 (84.4\%) & 451 (84.9\%) & .696 \\
\hline Diabetes presence & 1585 (48.9\%) & $224(42.2 \%)$ & $.001^{*}$ \\
\hline Coronary artery disease presence & $273(8.4 \%)$ & $26(4.9 \%)$ & $.001^{*}$ \\
\hline Cancer presence & $94(2.9 \%)$ & $2(0.4 \%)$ & $<.001^{*}$ \\
\hline Inability to ambulate presence & $198(6.1 \%)$ & $6(1.1 \%)$ & $<.001^{*}$ \\
\hline \multicolumn{4}{|l|}{ Neighborhood ${ }^{a}$} \\
\hline POOR, AA & $1563(48.2 \%)$ & $231(43.5 \%)$ & \multirow[t]{4}{*}{$.022^{*}$} \\
\hline POOR, WHITE & $408(12.6 \%)$ & $60(11.3 \%)$ & \\
\hline $\mathrm{RICH}, \mathrm{AA}$ & $573(17.7 \%)$ & 105 (19.8\%) & \\
\hline RICH, WHITE & $700(21.6 \%)$ & $135(25.4 \%)$ & \\
\hline $\begin{array}{l}\text { Median age at dialysis initiation (in } \\
\text { years) }^{b}\end{array}$ & $55(46,63)$ & $49(39,57)$ & $<.001^{*}$ \\
\hline Median body mass index $\left(\mathrm{kg} / \mathrm{m}^{2}\right)^{\mathrm{b}, \mathrm{c}}$ & $28.2(23.8,34.1)$ & $28.7(24.6,34.1)$ & .072 \\
\hline Median hemoglobin level $(\mathrm{g} / \mathrm{dL})^{\mathrm{b}, \mathrm{d}}$ & $9.6(8.5,10.7)$ & $9.7(8.5,10.9)$ & .412 \\
\hline
\end{tabular}

TABLE 1 Patient characteristics for non-Hispanic African Americans and whites in Chicago who initiated dialysis between January 2005 and September 2009 , overall and by waitlist appearance

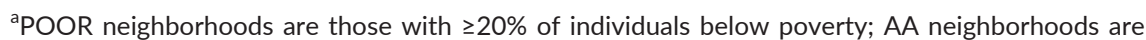
those with population $\geq 50 \%$ African American.

${ }^{\mathrm{b}} 25$ th and 75 th percentiles given in parentheses.

${ }^{\mathrm{c}} 216$ patients $(6.7 \%)$ were missing BMI.

${ }^{d} 335$ patients (10.3\%) were missing hemoglobin.

*Indicates a significant difference (at the 0.05 significance level) for waitlist appearance.

with our neighborhood variable as the explanatory variable of interest, adjusting for individual socioeconomic and clinical characteristics. All model-building and analyses were performed using Stata, version 14.0 (Stata Corp, College Station, TX, USA).

\section{3 | RESULTS}

Overall, of the 3244 patients in the study population, 531 patients (16.4\%) eventually appeared on the deceased donor kidney transplant waitlist during the observation period of the study. Patient characteristics for the entire study population and by waitlist appearance are shown in Table 1. The study population consisted of 2690 African American patients (82.9\%) and 554 white patients (17.1\%). The median age at a patient's first ESRD service was 55 years, and there were more male patients than female patients ( $55.7 \%$ vs $44.3 \%$ ). In addition, at dialysis initiation, 541 patients (16.7\%) did not have health insurance, and 1203 patients (37.1\%) were unemployed. Of the waitlisted patients, 440 were African American and 91 were white, each representing about $16.4 \%$ of their respective cohorts. The characteristics that were significantly different $(P<.05)$ with respect to waitlist appearance were medical insurance coverage, employment status, neighborhood, and the presence of diabetes, coronary artery disease, cancer, and inability to ambulate. There was also a significant difference $(P<.001)$ between the median age of those who were waitlisted and those who were not (49 vs 57 years old).

Patient characteristics by race are shown in Table 2. On average, African American patients were more likely to be uninsured (17.4\% vs $13.4 \%$ ), to have Medicaid as their primary medical insurance (35.9\% vs $17.5 \%$ ), or to have Medicare as their primary insurance (18.2\% vs $15.7 \%)$, compared to white patients. African Americans were also less likely to have private insurance ( $23.1 \%$ vs $37.4 \%$ ). In addition, African American patients were more likely to be unemployed $(40.2 \%$ vs $22.0 \%$ ) and tended to be younger (median age 55 vs 59), both $P<.05$. African American patients were less likely to be male than white patients (54.5\% vs 61.7\%); however, both races had a greater proportion of male patients than female patients. In terms of clinical characteristics, African American and white patients differed significantly in terms of hemoglobin count (median $9.5 \mathrm{vs} 9.9 \mathrm{~g} / \mathrm{dL}$ ), the presence of 
TABLE 2 Patient characteristics by race for non-Hispanic African Americans and whites in Chicago who initiated dialysis between January 2005 and September 2009

\begin{tabular}{|c|c|c|c|}
\hline & $\begin{array}{l}\text { African American } \\
\text { (2690 patients) }\end{array}$ & $\begin{array}{l}\text { White } \\
\text { (554 patients) }\end{array}$ & $P$-value \\
\hline Appearance on waitlist & $440(16.4 \%)$ & $91(16.4 \%)$ & .968 \\
\hline Male sex & $1466(54.5 \%)$ & $342(61.7 \%)$ & $.002^{*}$ \\
\hline \multicolumn{4}{|l|}{ Primary medical insurance } \\
\hline Uninsured & $467(17.4 \%)$ & $74(13.4 \%)$ & \multirow[t]{5}{*}{$<.001^{*}$} \\
\hline Medicaid & $966(35.9 \%)$ & $97(17.5 \%)$ & \\
\hline Medicare & 489 (18.2\%) & $87(15.7 \%)$ & \\
\hline Private insurance & $622(23.1 \%)$ & 207 (37.4\%) & \\
\hline Other insurance status & $146(5.4 \%)$ & $89(16.0 \%)$ & \\
\hline Unemployed & $1081(40.2 \%)$ & $122(22.0 \%)$ & $<.001^{*}$ \\
\hline Hypertension presence & 2307 (85.8\%) & $430(77.6 \%)$ & $<.001^{*}$ \\
\hline Diabetes presence & $1293(48.1 \%)$ & $292(52.7 \%)$ & $.047^{*}$ \\
\hline Coronary artery disease presence & $218(8.1 \%)$ & $55(9.9 \%)$ & .159 \\
\hline Cancer presence & $72(2.7 \%)$ & $22(4.0 \%)$ & .098 \\
\hline Inability to ambulate presence & $143(5.3 \%)$ & $55(9.9 \%)$ & $<.001^{*}$ \\
\hline \multicolumn{4}{|l|}{ Neighborhood $^{a}$} \\
\hline POOR, AA & $1526(56.7 \%)$ & $37(6.7 \%)$ & \multirow[t]{4}{*}{$<.001^{*}$} \\
\hline POOR, WHITE & 331 (12.3\%) & $77(13.9 \%)$ & \\
\hline $\mathrm{RICH}, \mathrm{AA}$ & $562(20.9 \%)$ & $11(2.0 \%)$ & \\
\hline RICH, WHITE & $271(10.0 \%)$ & 429 (77.4\%) & \\
\hline $\begin{array}{l}\text { Median age at dialysis initiation } \\
\text { (in years) }^{\text {b }}\end{array}$ & $55(45,62)$ & $59(52,65)$ & $<.001^{*}$ \\
\hline Median body mass index $\left(\mathrm{kg} / \mathrm{m}^{2}\right)^{\mathrm{b}, \mathrm{c}}$ & $28.3(23.7,34.2)$ & $27.4(24.4,33.6)$ & .336 \\
\hline Median hemoglobin level $(\mathrm{g} / \mathrm{dL})^{\mathrm{b}, \mathrm{d}}$ & $9.5(8.4,10.7)$ & $9.9(8.8,10.9)$ & $<.001^{*}$ \\
\hline
\end{tabular}

${ }^{a} \mathrm{POOR}$ neighborhoods are those with $\geq 20 \%$ of individuals below poverty; AA neighborhoods are those with population $\geq 50 \%$ African American.

${ }^{\mathrm{b}} 25$ th and 75 th percentiles given in parentheses.

${ }^{c} 216$ patients $(6.7 \%)$ were missing BMI.

$\mathrm{d}_{335}$ patients $(10.3 \%)$ were missing hemoglobin.

${ }^{*}$ Indicates a significant difference (at the 0.05 significance level) between African American and white. hypertension ( $85.8 \%$ vs $77.6 \%$ ) or diabetes ( $48.1 \%$ vs $52.7 \%$ ), and the inability to ambulate (5.3\% vs $9.9 \%$ ), all $P<.05$.

In terms of neighborhood characteristics, African American patients were significantly more likely to live in more disadvantaged neighborhoods (Table 2). About $69 \%$ of African American patients lived in ZIP codes with more than $20 \%$ of the population below poverty (POOR neighborhoods), compared to only $20.6 \%$ of white patients. African American patients also tended to live in neighborhoods that are predominantly African American, with $77.6 \%$ living in ZIP codes with an majority African American population (AA neighborhoods). Only $8.7 \%$ of white patients lived in ZIP codes with a majority African American population.

Our initial Cox proportional hazards model (Table 3) used race as the primary explanatory variable, adjusting for sex, insurance, employment status, hypertension, diabetes, coronary artery disease, cancer, inability to ambulate, age, BMI, and hemoglobin count. African American patients had a $27 \%$ lower cause-specific hazard of waitlist appearance than whites after adjusting for all other covariates (HR $0.73,95 \%[\mathrm{Cl}]$ 0.58, 0.92). Significant socioeconomic and clinical characteristics in our model were employment status, insurance status, age, cancer, and ambulatory status (all $P<.05$ ). Factors associated with an increased cause-specific hazard of waitlist include not being unemployed (HR 1.89 compared to unemployed) and having private insurance (HR 1.85 compared to uninsured patients), all $P<.05$. Factors associated with lower cause-specific hazard of transplant waitlist include cancer (HR 0.19 compared to those without cancer), inability to ambulate (HR 0.26 compared to those who are able to ambulate), and increasing age (HR 0.63 for each 10-year increase in age at first ESRD service). Testing the significance of the model as a whole, a $P$-value of $<.0001$ was reported, with a C-statistic of 0.703 . The proportional hazards assumption was not violated $(P=.316)$.

Our test for bias using interactions between the dummy variables and the variables with missing data (BMI and hemoglobin level) did not change the significance of any of the variables in our model. In addition, when we performed a likelihood ratio test (for nested models), there was not a significant difference between the model with the interactions and the model without the interactions (not shown).

Our second Cox model (Table 4) included a race by neighborhood interaction term. Compared to the reference group, white patients 
TABLE 3 Adjusted cause-specific hazard ratios from multivariable Cox model for time to deceased donor kidney transplant waitlist appearance (Model 1)

\begin{tabular}{|c|c|}
\hline & $\begin{array}{l}\text { Cause-specific hazard } \\
\text { ratio } \\
\text { ( } 95 \% \text { Confidence Interval) }\end{array}$ \\
\hline \multicolumn{2}{|l|}{ Race } \\
\hline White & Reference \\
\hline African American & $0.73(0.58-0.92)^{*}$ \\
\hline \multicolumn{2}{|l|}{ Sex } \\
\hline Male & Reference \\
\hline Female & $1.13(0.95-1.35)$ \\
\hline \multicolumn{2}{|l|}{ Primary medical insurance } \\
\hline Uninsured & Reference \\
\hline Medicaid & $0.84(0.65-1.10)$ \\
\hline Medicare & $0.98(0.69-1.38)$ \\
\hline Private insurance & $1.85(1.43-2.39)^{*}$ \\
\hline Other insurance status & $1.45(0.97-2.19)$ \\
\hline \multicolumn{2}{|l|}{ Employment status } \\
\hline Unemployed & Reference \\
\hline Other employment status & $1.89(1.55-2.29)^{*}$ \\
\hline \multicolumn{2}{|l|}{ Hypertension } \\
\hline No & Reference \\
\hline Yes & $1.05(0.83-1.34)$ \\
\hline \multicolumn{2}{|l|}{ Diabetes } \\
\hline No & Reference \\
\hline Yes & $0.90(0.75-1.08)$ \\
\hline \multicolumn{2}{|l|}{ Coronary artery disease } \\
\hline No & Reference \\
\hline Yes & $0.70(0.47-1.05)$ \\
\hline \multicolumn{2}{|l|}{ Cancer } \\
\hline No & Reference \\
\hline Yes & $0.19(0.05-0.78)^{*}$ \\
\hline \multicolumn{2}{|l|}{ Inability to ambulate } \\
\hline No & Reference \\
\hline Yes & $0.26(0.12-0.58)^{*}$ \\
\hline Age (per 10 y) & $0.63(0.59-0.68)^{*}$ \\
\hline Body mass index & $1.00(0.99-1.01)$ \\
\hline Hemoglobin level & $1.01(0.97-1.06)$ \\
\hline
\end{tabular}

*Indicates a significant difference (at the 0.05 significance level).

in RICH, WHITE neighborhoods, African Americans living in poor, or African American-majority neighborhoods had a significantly lower cause-specific hazard of waitlist appearance. African Americans living in POOR, AA neighborhoods had a $40 \%$ lower cause-specific hazard of waitlist appearance (HR 0.60, 95\% [Cl] 0.44, 0.84), African Americans living in POOR, WHITE neighborhoods had a $41 \%$ lower cause-specific hazard (HR 0.59, 95\% [Cl] 0.40, 0.85), and African Americans living in $\mathrm{RICH}, \mathrm{AA}$ neighborhoods had a $31 \%$ lower cause-specific hazard (HR $0.69,95 \%[\mathrm{Cl}] 0.50,0.97)$. Compared to the reference group, there was no significant difference for white patients in any neighborhood category nor for African Americans in RICH, WHITE neighborhoods. All other significant covariates in Model 1 remained significant in this model. A C-statistic of 0.705 was calculated for this model, suggesting a slight increase in predictive power compared to the first model. The proportional hazards assumption was not violated $(P=.180)$.

When we compared Model 1 with individual race and no neighborhood-level covariates to a new model with race and our neighborhood variable as separate predictors, race was no longer statistically significant in the new model and the white-African American hazard ratio changed from 0.73 (in Model 1) to 0.94 (analysis not shown). To quantify the effect of neighborhood, our calculation estimated that $(0.94-0.73) /(1-0.73) \approx 0.778$, or $77.8 \%$, of racial disparities in Chicago can be explained by neighborhood poverty and racial composition.

Table 5 shows the adjusted cause-specific hazard ratios with only a race by neighborhood poverty interaction or a race by neighborhood racial composition interaction, adjusting for sociodemographic and clinical characteristics. When looking at neighborhood poverty, African Americans in poor (POOR) neighborhoods are the only group with a significantly lower cause-specific hazard ratio compared to the reference group, white patients in $\mathrm{RICH}$ neighborhoods (HR 0.61, 95\% [Cl] 0.44, 0.84). Similarly, when looking at neighborhood racial composition, African Americans in predominately African American (AA) neighborhoods have a significantly lower cause-specific hazard ratio than the reference group, white patients in predominately white (WHITE) neighborhoods (HR 0.66, 95\% [Cl] 0.50, 0.88).

We then constructed separate Cox models stratified by race using our neighborhood variable as the explanatory variable of interest, adjusting for individual socioeconomic and clinical characteristics to examine if neighborhood characteristics affect whites and African Americans differently (analysis not shown). For whites, patients in any neighborhood category did not have a significantly different cause-specific hazard of waitlist appearance compared to patients in RICH, WHITE neighborhoods. For African Americans, patients in POOR neighborhoods were significantly less likely to be waitlisted than patients in RICH, WHITE neighborhoods (POOR, AA HR 0.67, 95\% [Cl] 0.46, 0.95 and POOR, WHITE HR 0.65, 95\% [Cl] 0.44, 0.97). Looking only at neighborhood poverty, African Americans in POOR neighborhoods had a $21 \%$ lower cause-specific hazard of waitlist compared to African Americans in $\mathrm{RICH}$ neighborhoods (HR 0.79, 95\% [Cl] 0.64, 0.98).

\section{4 | DISCUSSION}

We found that there are significant racial disparities in the likelihood of reaching the kidney transplant waitlist in Chicago. African American patients in Chicago are significantly less likely than white patients to appear on the renal transplant waitlist (HR 0.73) even after adjusting for other sociodemographic and clinical factors. This is consistent with our prior work, which showed that in UNOS region 7, which contains the state of Illinois, African Americans had a 22\% lower hazard of waitlist appearance than whites. ${ }^{9}$ 
TABLE 4 Adjusted cause-specific hazard ratios for waitlist appearance by race and neighborhood composition (Model 2)

\begin{tabular}{llll}
$\begin{array}{lll}\text { Neighborhood racial } \\
\text { majority }\end{array}$ & Patient race & RICH & POOR \\
\cline { 3 - 4 } WHITE & White & Reference & $0.76(0.45-1.28)$ \\
& African American & $0.90(0.61-1.34)$ & $0.59(0.40-0.85)^{*}$ \\
AA & White & $0.51(0.08-3.44)$ & $0.27(0.07-1.10)$ \\
& African American & $0.69(0.50-0.97)^{*}$ & $0.60(0.44-0.84)^{*}$
\end{tabular}

Adjusted for sociodemographic (race, sex, insurance coverage, employment status, age) and clinical characteristics (hypertension, diabetes, coronary artery disease, cancer, ambulatory status, BMI, and hemoglobin level).

*Indicates a significant difference (at the 0.05 significance level).

TAB LE 5 Adjusted cause-specific hazard ratios for waitlist appearance by race and poverty/race and racial composition alone

\begin{tabular}{llllll} 
& \multicolumn{2}{l}{ Neighborhood poverty } & & & \multicolumn{2}{l}{ Neighborhood racial majority } \\
\cline { 2 - 3 } Patient race & RICH & POOR & & WHITE & AA \\
White & Reference & $0.62(0.36-1.07)$ & & Reference & $0.34(0.11-1.07)$ \\
African American & $0.77(0.56-1.04)$ & $0.61(0.44-0.84)^{*}$ & & $0.77(0.55-1.06)$ & $0.66(0.50-0.88)^{*}$ \\
\hline
\end{tabular}

Adjusted for sociodemographic (race, sex, insurance coverage, employment status, age) and clinical characteristics (hypertension, diabetes, coronary artery disease, cancer, ambulatory status, BMI, and hemoglobin level).

*Indicates a significant difference (at the 0.05 significance level).

We also found that the extent of the disparities depends on neighborhood-level characteristics. Both neighborhood racial composition and neighborhood poverty are associated with racial disparities in transplant waitlist for African Americans. When we examined patients both by individual race and by neighborhood poverty and racial composition, we see a significant disparity for African Americans living in poor or predominantly African American neighborhoods, but not for African Americans in nonpoor, predominantly white neighborhoods. African Americans in poor neighborhoods, regardless of racial composition, have a significantly lower cause-specific hazard of transplant waitlist appearance compared to whites in rich neighborhoods (HR 0.61 overall). African Americans in predominantly African American neighborhoods, regardless of neighborhood poverty, are also less likely to appear on the transplant waitlist compared to whites in predominantly white neighborhoods (HR 0.66 overall). In addition, African Americans in poor neighborhoods were significantly less likely to appear on the transplant waitlist than their African American counterparts in wealthier neighborhoods (HR 0.79). Our finding is particularly salient given that over $69 \%$ of African Americans in our study population live in high-poverty neighborhoods, compared to only $21 \%$ of whites. Whites did not vary significantly in access to the transplant waitlist in any neighborhood classification. We estimate that in Chicago approximately $78 \%$ of racial disparities in access to the renal transplant waitlist are associated with neighborhood poverty and racial composition. Our work is consistent with previous work that demonstrates that neighborhood composition, especially neighborhood poverty, is associated with lower access to transplantation. ${ }^{10,11,14}$

Older age, having a history of cancer, inability to ambulate, and being unemployed are associated with a significantly lower hazard of transplant waitlist appearance. Compared to being uninsured, having private insurance is significantly associated with a greater likelihood of waitlist appearance. These results are consistent with previous literature on kidney transplant waitlist access. $5,18,19,23-26$

During the era we examined, individuals accrued waitlist time only after transplant waitlist candidacy. Thus, delays in reaching transplant waitlist increased pretransplant dialysis time, which is associated with both decreased graft and patient survival. ${ }^{27}$ In 2014, UNOS changed the kidney allocation system to allow individuals to receive credit for time they spent on dialysis prior to waitlisting. ${ }^{28}$ The results of our study underscore the need for such a policy change. Recent work has shown that the kidney allocation system has reduced racial disparities to transplant access nationally. ${ }^{29}$ To date, the impact of the policy on local- or regional-level disparities has been less well examined.

There are several limitations to our study. The first is limited data on patient socioeconomic status, including income, individual-level poverty, or social support. Thus, we were unable to include these factors in our model, even though it has been suggested that these socioeconomic factors help explain some of the racial disparities associated with kidney transplantation waitlisting. ${ }^{26}$ Prior work has demonstrated that community-level factors exert an independent effect on transplant processes even after controlling for patient SES (education and insurance) and clinical factors. ${ }^{30}$ In addition, we also did not have data regarding a patient's preferences regarding transplantation, another factor affecting waitlist access. ${ }^{8}$ Another potential source of error is the limited data regarding comorbid conditions from the medical evidence form including the absence of information related to disease severity and inadequate sensitivity for certain conditions. ${ }^{31}$ Furthermore, these forms were collected at dialysis initiation, so our model could not adequately account for changes in the patient's health status over time. However, we do not believe that change of health over time would introduce a significant bias to our results unless it occurred in a systematic manner. 
Our imputation method for missing data also has the potential to generate some degree of bias, as adding a dummy variable to indicate missing data tends to cause the standard error of estimates to be lower than what their true values should be, which in turn may cause $P$-values in the full model to be smaller than what they should be. Our two tests for bias, examining for interaction between the dummy variables and the variables with missing data (BMI and hemoglobin level) and performing a likelihood ratio test (for nested models), did not demonstrate significance of the imputed data. Thus, we do not believe that the bias caused by our imputation methods significantly affected our results, and we proceeded with our original models without the dummy interaction terms.

An additional limitation is that there were very few white patients who lived in African American neighborhoods, which caused a large standard error in our second model for white patients. Finally, because our analyses were based only on the city of Chicago, it may be difficult to generalize our results to other cities and regions. Racial composition and spatial dynamics may vary by city or region. However, we believe that Chicago serves as an ideal case study to examine neighborhood effects on disparities due to its large ESRD population and its continued neighborhood segregation by race and income. ${ }^{15,16}$

Finally, our work is unable to determine the causes of reduced waitlist access for African Americans in poor neighborhoods and predominantly African American neighborhoods. Prior work has shown that African Americans and poor individuals are less likely to be deemed appropriate candidates for transplant or referred for transplantation even when medically appropriate. ${ }^{8,22}$ This may be due to healthcare providers' unconscious bias about poor, minority patients desire or suitability for transplant. In addition, African Americans often receive care close to where they live. ${ }^{32}$ Dialysis facility providers and staff in poor, minority communities may not be as well informed about transplant or be connected to a transplant center. ${ }^{33}$ Residents of poor, minority areas may have lower levels of social capital and weaker social networks, which may lead to a lack of information about transplantation, unreliable transportation to appointments, or fewer caretakers who could assist them in navigating the transplant process. ${ }^{11,34}$ Thus, these individuals may take a longer time to navigate the extensive pretransplant medical and psychosocial evaluation or may be more likely to have relative financial and social contraindications to transplant. ${ }^{11,35}$ Several interventions have been developed to reduce transplant disparities and improve access to transplant for African Americans. Disparity reduction strategies include patient-level strategies such as patient education and patient navigators as well as system-level changes at the dialysis facility or at the policy level. ${ }^{29,36-39}$

Our study finds racial disparities in access to the renal transplantation waitlist in Chicago and that the extent of these disparities depends on neighborhood-level characteristics. Our finding is important because in Chicago, and likely nationwide, neighborhood poverty impacts African Americans more severely than other groups, and in addition, a higher proportion of African Americans live in highpoverty neighborhoods. ${ }^{40}$ However, African Americans in wealthier (nonpoor) African American neighborhoods were still less likely to appear on the transplant waitlist compared to their counterparts in wealthier white neighborhoods. Additional interventions targeted to both race and neighborhood are needed to increase equity of access to renal transplantation. The complete picture of racial disparities in kidney transplantation access likely depends on a combination of patient, provider, neighborhood, and regional factors. Gaining a better understanding of the underlying causes and nature of these observed disparities is a vital step to take in order to develop methods that will help improve equality in kidney transplantation access and overcome barriers to quality health care.

\section{CONFLICT OF INTEREST}

None.

\section{ORCID}

Milda R. Saunders (iD http://orcid.org/0000-0002-9276-2066

\section{REFERENCES}

1. 2016 USRDS annual data report: Epidemiology of kidney disease in the United States. Bethesda, MD. 2016.

2. Tonelli M, Wiebe N, Knoll G, et al. Systematic review: kidney transplantation compared with dialysis in clinically relevant outcomes. Am J Transplant. 2011;11:2093-2109.

3. Alexander GC, Sehgal AR. Barriers to cadaveric renal transplantation among blacks, women, and the poor. JAMA. 1998;280: 1148-1152.

4. Goldfarb-Rumyantzev AS, Sandhu GS, Baird B, et al. Effect of education on racial disparities in access to kidney transplantation. Clin Transplant. 2012;26:74-81.

5. Wolfe RA, Ashby VB, Milford EL, et al. Differences in access to cadaveric renal transplantation in the United States. Am J Kidney Dis. 2000;36:1025-1033.

6. Kucirka LM, Purnell TS, Segev DL. Improving access to kidney transplantation: referral is not enough. JAMA. 2015;314:565-567.

7. Garg PP, Diener-West M, Powe NR. Reducing racial disparities in transplant activation: whom should we target? Am J Kidney Dis. 2001;37:921-931.

8. Ayanian JZ, Cleary PD, Keogh JH, Noonan SJ, David-Kasdan JA, Epstein AM. Physicians' beliefs about racial differences in referral for renal transplantation. Am J Kidney Dis. 2004;43:350-357.

9. Saunders MR, Lee H, Alexander GC, Tak HJ, Thistlethwaite JR, Ross LF. Racial disparities in reaching the renal transplant waitlist: is geography as important as race? Clin Transplant. 2015;29:531-538.

10. Saunders M, Cagney K, Ross L, Alexander G. Neighborhood poverty, racial composition and renal transplant waitlist. Am J Transplant. 2010;10:1912-1917.

11. Joshi S, Gaynor JJ, Bayers S, et al. Disparities among Blacks, Hispanics, and Whites in time from starting dialysis to kidney transplant waitlisting. Transplantation. 2013;95:309-318.

12. Mathur A, Ashby V, Sands R, Wolfe R. Geographic variation in endstage renal disease incidence and access to deceased donor kidney transplantation. Am J Transplant. 2010;10:1069-1080.

13. Tonelli M, Klarenbach S, Manns B, et al. Residence location and likelihood of kidney transplantation. CMAJ. 2006;175:478-482.

14. Patzer RE, Amaral S, Wasse H, Volkova N, Kleinbaum D, McClellan WM. Neighborhood poverty and racial disparities in kidney transplant waitlisting. J Am Soc Nephrol. 2009;20:1333-1340. 
15. Sandoval JO. Neighborhood diversity and segregation in the Chicago metropolitan region, 1980-2000. Urban Geogr. 2011;32: 609-640.

16. 2007-2011 American Community Survey 5-Year Estimates. Chicago, IL. 2011.

17. Grams ME, Massie AB, Schold JD, Chen B, Segev DL. Trends in the inactive kidney transplant waitlist and implications for candidate survival. Am J Transplant. 2013;13:1012-1018.

18. Sandhu GS, Khattak M, Pavlakis $M$, et al. Recipient's unemployment restricts access to renal transplantation. Clin Transplant. 2013;27:598-606.

19. Johansen KL, Zhang R, Huang Y, Patzer RE, Kutner NG. Association of race and insurance type with delayed assessment for kidney transplantation among patients initiating dialysis in the United States. Clin J Am Soc Nephrol. 2012;7:1490-1497.

20. Poverty Areas. 1995. https://www.census.gov/population/socdemo/ statbriefs/povarea.html 2017. Accessed April 14, 2017.

21. Austin PC, Lee DS, Fine JP. Introduction to the analysis of survival data in the presence of competing risks. Circulation. 2016;133:601-609.

22. Patzer RE, Perryman JP, Schrager JD, et al. The role of race and poverty on steps to kidney transplantation in the southeastern United States. Am J Transplant. 2012;12:358-368.

23. Weng FL, Reese PP, Mulgaonkar S, Patel AM. Barriers to living donor kidney transplantation among black or older transplant candidates. Clin J Am Soc Nephrol. 2010;5:2338-2347.

24. Rhee CM, Lertdumrongluk P, Streja E, et al. Impact of age, race and ethnicity on dialysis patient survival and kidney transplantation disparities. Am J Nephrol. 2014;39:183-194.

25. Hall YN, Choi Al, Xu P, O'Hare AM, Chertow GM. Racial ethnic differences in rates and determinants of deceased donor kidney transplantation. J Am Soc Nephrol. 2011;22:743-751.

26. Patzer R, Amaral S, Klein M, et al. Racial disparities in pediatric access to kidney transplantation: does socioeconomic status play a role? Am J Transplant. 2012;12:369-378.

27. Meier-Kriesche HU, Schold JD. The impact of pretransplant dialysis on outcomes in renal transplantation. Semin Dial. 2005;18: 499-504.

28. The New Kidney Allocation System (KAS) Frequently Asked Questions [Webpage]. 2017. https://optn.transplant.hrsa.gov/media/1235/kas faqs.pdf. Accessed November 28, 2017.

29. Melanson TA, Hockenberry JM, Plantinga L, et al. New kidney allocation system associated with increased rates of transplants among black and Hispanic patients. Health Aff. 2017;36:1078-1085.

30. Schold JD, Heaphy ELG, Buccini LD, et al. Prominent impact of community risk factors on kidney transplant candidate processes and outcomes. Am J Transplant. 2013;13:2374-2383.
31. Longnecker JC, Coresh J, Klag MJ, et al. Validation of comorbid conditions on the end-stage renal disease medical evidence report the CHOICE study. J Am Soc Nephrol. 2000;11:520-529.

32. Saunders MR, Lee H, Maene C, Schuble T, Cagney KA. Proximity does not equal access: racial disparities in access to high quality dialysis facilities. J Racial Ethn Health Disparities. 2014;1:291-299.

33. Plantinga LC, Pastan SO, Wilk AS, et al. Referral for kidney transplantation and indicators of quality of dialysis care: a cross-sectional study. Am J Kidney Dis. 2017;69:257-265.

34. Clark CR, Hicks LS, Keogh JH, Epstein AM, Ayanian JZ. Promoting access to renal transplantation: the role of social support networks in completing pre-transplant evaluations. J Gen Intern Med. 2008;23:1187.

35. Lockwood MB, Saunders MR, Nass R, et al. Patient-reported barriers to the prekidney transplant evaluation in an at-risk population in the United States. Prog Transplant. 2017;27:131-138.

36. Sullivan C, Leon JB, Sayre SS, et al. Impact of navigators on completion of steps in the kidney transplant process: a randomized, controlled trial. Clin J Am Soc Nephrol. 2012;7:1639-1645.

37. Waterman AD, McSorley AM, Goalby CJ, et al. Explore transplant at home: a randomized control trial of an educational intervention to increase transplant knowledge for black and white socioeconomically disadvantaged dialysis patients. BMC Nephrol. 2015;16:150.

38. Patzer RE, Perryman JP, Pastan S, et al. Impact of a patient education program on disparities in kidney transplant evaluation. Clin J Am Soc Nephrol. 2012;7:648-655.

39. Patzer RE, Paul S, Plantinga L, et al. A randomized trial to reduce disparities in referral for transplant evaluation. J Am Soc Nephrol. 2017;28:935-942.

40. Firebaugh G, Farrell CR. Still large, but narrowing: the sizable decline in racial neighborhood inequality in metropolitan America, 19802010. Demography. 2016;53:139-164

\section{SUPPORTING INFORMATION}

Additional Supporting Information may be found online in the supporting information tab for this article.

How to cite this article: Peng RB, Lee H, Ke ZT, Saunders MR.

Racial disparities in kidney transplant waitlist appearance in chicago: Is it race or place?. Clin Transplant. 2018;32:e13195. https://doi.org/10.1111/ctr.13195 